\title{
Capacidade institucional dos serviços de saúde antes, durante e após a implantação do Modelo de Atenção às Condições Crônicas (MACC)
}

\author{
Institutional capacity of health services before, during and after the implementation of a \\ model for chronic care conditions (MACC)
}
Capacidad institucional de los servicios de salud antes, durante y después de la implementación de un Modelo de Atención a Condiciones Crónicas (MACC)

Cláudia Ferreira Melo Rodrigues ${ }^{1,2 *}$, Clareci Silva Cardoso ${ }^{1,2}$, Nayara Ragi Baldoni ${ }^{1,3}$, Thays Aparecida Leão D'Alessandro ${ }^{1}$, Nayara Dornela Quintino ${ }^{1,2}$, Kenya Valeria Micaela de Souza Noronha $^{4}$, Laíse Oliveira Resende ${ }^{1,2}$, Monica Viegas Andrade ${ }^{4}$.

\section{RESUMO}

Objetivo: Avaliar a capacidade institucional dos serviços de saúde na percepção dos profissionais no contexto da implantação do Modelo de Atenção às Condições Crônicas explorando as diferenças entre as unidades básicas de saúde. Métodos: Trata-se de um estudo de painel com avaliação antes (2013), durante (2015) e após (2018) a implantação do Modelo de Atenção às Condições Crônicas (MACC) no sistema de saúde com foco em três condições crônicas: Diabetes Mellitus, Hipertensão e gestantes. Nos três momentos, os profissionais de saúde das unidades da atenção primária e especializada avaliaram a capacidade institucional por meio do instrumento Assessment of Chronic Illness Care (ACIC). Resultados: Após a implantação do MACC observa-se um aumento estatisticamente significativo dos escores do ACIC para todas as dimensões em todas unidades de saúde. Conclusão: $\mathrm{Na}$ avaliação dos profissionais foi evidenciado ganho importante na capacidade institucional do município para o cuidado das condições crônicas após intervenção no sistema de saúde.

Palavras-chave: Condição crônica, Avaliação em saúde, Atenção primária à saúde, Assistência à saúde.

\section{ABSTRACT}

Objective: To evaluate the institutional capacity of health services in the professionals' perception inside the context of ther Chronic Conditions Care Model implementation, exploring the differences between basic health units. Methods: This work concerns a panel study with evaluation before (2013), during (2015) and after (2018) the implementation of the Chronic Conditions Care Model (CCCM) in the health system, focusing on three chronic conditions: Diabetes Mellitus, Hypertension and pregnant women. Among the three moments, health professionals from primary and specialized care units assessed institutional capacity using the Assessment of Chronic Illness Care (ACIC) instrument. Results: After the CCCM implantation, it is observed a statistically significant increase in the ACIC scores for all the dimensions in all health units. Conclusion: Towards the evaluation of the professionals, an important gain was pointed out in the municipality institutional capacity to care for chronic conditions after intervention in the health system.

Keywords: Chronic condition, Health evaluation, Primary health care, Health care.

\footnotetext{
${ }^{1}$ Grupo de Pesquisa em Epidemiologia e Avaliação de Novas Tecnologias em Saúde, Divinópolis - MG.

2 Universidade Federal de São João del-Rei (UFSJ), Divinópolis - MG.

*E-mail:melo.claudia@hotmail.com

3 Universidade de Itaúna (UIT), Itaúna - MG.

${ }^{4}$ Universidade Federal de Minas Gerais (UFMG), Belo Horizonte - MG.
} 


\section{RESUMEN}

Objetivo: Evaluar la capacidad institucional de los servicios de salud en la percepción de los profesionales en el contexto de la implantación del Modelo de Atención a las Condiciones Crónicas explorando las diferencias entre las unidades básicas de salud. Métodos: Se trata de un estudio de painel con evaluación antes (2013), durante (2015) y después (2018) la implantación del Modelo de Atención a las Condiciones Crónicas (MACC) en el sistema de salud con foco en tres condiciones crónicas: Diabetes Mellitus, Hipertensión y gestantes. En los tres momentos, los profesionales de salud de las unidades de la atención primaria y especializada evaluaron la capacidad institucional por medio del instrumento Assessment of Chronic Illness Care (ACIC). Resultados: Tras la implantación del MACC se observa un aumento estadísticamente significativo de los escores del ACIC para todas las dimensiones en todas las unidades de salud. Conclusión: En la evaluación de los profesionales se evidenció un logro importante en la capacidad institucional del municipio para el cuidado de las condiciones crónicas tras intervención en el sistema de salud.

Palabras clave: Condición crónica, Evaluación en salud, Atención primaria a la salud, Asistencia a la salud.

\section{INTRODUÇÃO}

A Rede de Atenção à Saúde (RAS) surgiu com o objetivo de construir um sistema integrado de ações e serviços de saúde com provisão de atenção contínua, integral, de qualidade, responsável e humanizada, visando superar a fragmentação da atenção à saúde (MENDES EV, 2011; D’ÁVILA ALV, et al., 2018). Implantar RAS efetivas é um grande desafio considerando o atual perfil epidemiológico, no qual, as doenças crônicas não transmissíveis (DCNT) são responsáveis por aproximadamente $70 \%$ das mortes no mundo (SCHMIDT MI, et al., 2011).

No Brasil, a carga das DCNT é responsável por $66,3 \%$ dos anos de vida perdidos ajustados por incapacidade, o que compromete a qualidade de vida das pessoas além dos custos econômicos, sendo estimados em US\$ sete trilhões os gastos com DCNT, durante os anos de 2011-2025, em países de baixa e média renda (SCHRAMM JMA, et al., 2004; ABEGUNDE DO, et al., 2007; MALTA DC, et al., 2017). Diante desse cenário nota-se a importância de uma RAS atuante no cuidado às condições crônicas (CC).

No Brasil, uma das estratégias utilizadas para o enfrentamento das CC tem sido a implementação de intervenções no sistema de saúde. Um exemplo é o Modelo de Atenção às Condições Crônicas (MACC), cuja proposta é a qualificação da atenção primária à saúde (APS) para o cuidado às CC integrando as ações de promoção da saúde, prevenção de doenças e qualificação de linhas de cuidado (MENDES EV, et al., 2018; MOYSÉS ST, et al., 2012).

Nesse contexto, a assistência em saúde é organizada sobretudo a partir dos princípios da territorialização, classificação e estratificação do risco que estão alinhados com a lógica de organização da Atenção Primária (ANDRADE MV, et al., 2019). Cenário que envolve alterações nas condutas dos profissionais e mudanças nos processos de trabalho, assim como na coordenação do cuidado. O MACC contribui com soluções práticas e inovadoras para o cuidado das $\mathrm{CC}$ e essenciais para prestar um atendimento adequado, longitudinal e em Rede (EVANGELISTA MJO, et al., 2019).

Há evidencias na literatura sobre a contribuição positiva do MACC, como pode ser observado no estudo de coorte retrospectivo de base populacional realizado em Puglia, Itália. Tal trabalho identificou reduções de dias, bem como, de custos de internações em um grupo de pessoas com condições CC incluídas em um programa de cuidados orientados pelo Chronic Care Model (CCM), quando comparado com indivíduos que receberam atendimentos habituais (ROBUSTO F, et al., 2018).

A eficiência do MACC também foi mencionada em uma revisão sistemática realizada por Davy $C$, et al. (2015), onde 75 artigos mostraram melhorias na prática de saúde com pessoas que vivem com CC. Tal eficiência corrobora com outra revisão sistemática realizada por Baptista DR, et al. (2016), a qual identificou que, embora ações isoladas sejam ineficazes no tratamento do diabetes Mellitus (DM), o uso conjunto dos seis elementos do CCM possibilita a obtenção de resultados benéficos no tratamento de tal condição crônica. 
O presente estudo aborda a implementação do MACC no sistema de saúde em um município de Minas Gerais, a primeira intervenção em base populacional no Brasil. O objetivo do trabalho é analisar a capacidade institucional dos serviços de saúde na percepção dos profissionais no contexto da implantação do MACC explorando as diferenças entre as unidades básicas de saúde, antes, durante e após a intervenção. Essa abordagem é fundamental uma vez que permite inferir se as mudanças na capacidade institucional foram permanentes ou se foram condicionadas a presença de atores externos durante o período da intervenção.

\section{MÉTODOS}

\section{Princípios éticos}

Este estudo foi aprovado pelo comitê de ética em pesquisa da Universidade Federal de São João del-Rei (UFSJ) sob o parecer 369.942/2013.

\section{Delineamento e local do estudo}

Trata-se de um estudo do tipo painel com avaliação antes, durante e após uma intervenção. Esta investigação ocorreu em um município mineiro, com estimativa da população em 2019 de 28.243 habitantes (IBGE, 2019). O presente estudo é representativo de toda APS do município, incluindo as oito Unidades Básicas de Saúde e o Centro de Atenção Especializada, representados pelas letras A até I.

\section{Intervenção: implantação do MACC}

A intervenção do MACC ocorreu com foco na organização da assistência para três CC: DM, hipertensão arterial sistêmica (HAS) e gestantes e incluiu os profissionais de todas as UBS, urbanas e rurais. O objetivo do MACC foi o fortalecimento da APS para atuação nessas condições por meio da implementação de protocolos e ferramentas inovadoras que buscavam garantir um atendimento longitudinal e integrado. Para atingir tal objetivo, a intervenção seguiu etapas propostas pelo referencial teórico MACC para organização dos macroprocessos da APS (ANDRADE MV, et al., 2019).

\section{Avaliação e instrumentos de coleta dados}

A avaliação da intervenção no sistema de saúde foi realizada por profissionais da APS e da atenção secundária, e foi baseada na mudança de processos de trabalho que ocorreu em três momentos, distintos: a) na linha de base, antes da intervenção do MACC (2013), b) ao final da intervenção (2015) e, c) após três anos (2018).

Os grupos focais contaram com a participação de um representante de cada categoria profissional com atuação no serviço. Os profissionais deveriam ter experiência mínima de 12 meses e os médicos, atuação mínima de seis meses no serviço de saúde do município. Quando existia mais de um profissional elegível para essa avaliação foi feito sorteio dentro de cada categorial profissional, pois cada grupo deveria ter no máximo dez participantes.

Os participantes selecionados responderam ao instrumento de Avaliação da Capacidade Institucional, Assessment of Chronic Illness Care (ACIC), validado para o Brasil por Moysés ST, et al. (2012). O ACIC é composto por 35 questões norteadoras divididas em sete dimensões: Dimensão 1 - Organização da Atenção à Saúde; Dimensão 2 - Articulação com a comunidade; Dimensão 3 - Autocuidado apoiado; Dimensão 4 Suporte à decisão; Dimensão 5 - Desenho do sistema de prestação de serviço; Dimensão 6 - Sistema de informação clínica; Dimensão 7: Integração dos componentes do MACC.

Estas dimensões recebem pontuação na escala Likert de 0 a 11. Obtendo-se uma pontuação para cada dimensão, que ao final, são somadas e divididas pelo número de dimensões resultando na Capacidade Institucional, a qual é classificada em quatro níveis de atenção às CC: resultados entre 0 e 2 (limitada: nível D); entre 3 e 5 (básica: nível C); entre 6 e 8 (razoável: nível B); entre 9 e 11 (ótima: nível A).

A avaliação foi conduzida por meio da discussão em grupo, baseando-se no referencial teórico de grupos focais e, coordenados por psicólogos (GILL P, et al., 2008). Embora a discussão do ACIC tenha sido realizada em grupo, o preenchimento do mesmo foi realizado individualmente. 
O instrumento foi aplicado por meio da discussão em nove grupos, sendo constituído um grupo para cada unidade de saúde. Além da avaliação da implantação, realizou-se um levantamento do perfil sociodemográfico dos usuários, bem como o acesso ao serviço de saúde. Essas informações foram coletadas na linha de base do estudo.

Para avaliar o perfil dos pacientes com condições crônicas segundo as Unidades Básicas de Saúde foram utilizadas as informações obtidas em pesquisa de campo domiciliar de base populacional realizada em dois recortes transversais 2013 e 2015. Os pacientes foram identificados como portadores de doenças crônicas por meio de auto relato dos entrevistados. Maiores detalhes sobre essa pesquisa de campo podem ser obtidos em Andrade MV, et al. (2019).

\section{Análise de dados}

Os dados sócios demográficos foram analisados por meio de estatística descritiva. Foram utilizados os testes não paramétricos de kruskall wallis, para detectar diferenças entre os três momentos da Avaliação da Capacidade Institucional avaliadas pelo instrumento ACIC.

\section{RESULTADOS}

Dentre os usuários, portadores de doenças crônicas, que utilizam o serviço de saúde do município, 832 participaram da linha de base. Em relação ao perfil dos usuários nota-se heterogeneidade socioeconômica nos indicadores de classe social e escolaridade. As unidades rurais possuem maior proporção de indivíduos nas classes D-E e com níveis de escolaridade até a quarta série do ensino fundamental (Tabela 1).

Quanto o acesso aos serviços de atenção primária a saúde nota-se cobertura total do munícipio em relação ao cadastro dos domicílios em todas as UBS e em relação a utilização de serviços. Observa-se também que o serviço mais utilizado pelos usuários do município é a APS, sendo esta uma referência no município.

Mesmo com elevada cobertura dos serviços públicos de saúde, observa-se cobertura de plano privado importante (34,9\%), superior, à cobertura do estado (20\% a $30 \%)$ e do Brasil $(24,1 \%)$ (BRASIL, 2020). Entre as Unidades Básicas de Saúde a cobertura de plano privado apresenta grande variação, de 17\% na UBS C a $49 \%$ na UBS G. As UBS com maior cobertura de plano privado são as que os usuários apresentam as melhores condições socioeconômicas (Tabela 2).

A tabela 3 mostra as características sociodemográficas e tempo de inserção no serviço dos profissionais de saúde do município que responderam ao ACIC. Nos três anos, a maioria dos profissionais era do sexo feminino e tinha pelo menos ensino médio. Em relação ao tempo de atuação, para praticamente todas as UBS comparando antes (2013) e depois da intervenção (2018) percebe-se um aumento da proporção de profissionais com mais de seis anos de serviço (Tabela 3 ).

Na tabela 4 estão apresentados os escores da Capacidade Institucional do sistema de saúde por unidade de saúde nos três momentos da avaliação. De forma geral, observa-se um aumento dos escores do ACIC em todas as dimensões para todas as unidades de saúde, com diferença estatisticamente significativa $(p<0,01)$.

Ao se analisar a avaliação global do ACIC por unidade básica de saúde observa-se aumento dos escores para todas as unidades exceto para a $\mathrm{D}$ que embora tenha apresentado um aumento, sua capacidade global manteve-se classificada como razoável nos três momentos do estudo. Importante mencionar que seis das nove unidades avaliadas alcançaram capacidade ótima em 2018, seis anos após a intervenção pelo MACC (Tabela 4).

Ao fazer análise por dimensão ao longo dos anos observa-se que algumas unidades dobraram ou triplicaram seus escores ao comparar antes e após a intervenção, na Dimensão 6 (Sistema de Informação Clínica) e na Dimensão 7 (Integração dos componentes do MACC).

Estas dimensões possuíam baixa avaliação em 2013, mas melhoraram significativamente seus escores em 2018. Entretanto, a Dimensão 2 (Articulação com a comunidade) foi a que apresentou menor evolução ao longo dos anos nas unidades $A, D, F$ e G, com destaque para as unidades $A$ e $D$ cujas avaliações diminuíram em $33 \%$ e $9 \%$, respectivamente. 
Tabela 1 - Perfil sociodemográfico dos usuários do sistema público de saúde no inquérito domiciliar, n=832.

\begin{tabular}{|c|c|c|c|c|c|c|c|c|}
\hline \multirow{3}{*}{ Variável } & \multicolumn{8}{|c|}{ Unidades de Saúde } \\
\hline & A & B & C & D & $\mathbf{E}$ & $\mathbf{F}$ & $\mathbf{G}$ & $\mathbf{H}$ \\
\hline & n (\%) & n (\%) & n (\%) & n (\%) & n (\%) & n (\%) & n (\%) & n (\%) \\
\hline Total & $145(100)$ & $125(100)$ & $138(100)$ & $114(100)$ & $34(100)$ & $49(100)$ & $96(100)$ & $131(100)$ \\
\hline \multicolumn{9}{|l|}{ Sexo } \\
\hline Feminino & $92(63,4)$ & $79(63,2)$ & $98(71)$ & $88(77,2)$ & $21(61,8)$ & $38(77,6)$ & $65(67,7)$ & $92(70,2)$ \\
\hline Idade: média (土DP) & $57,4(16,6)$ & $50,6(19,2)$ & $48,0(15,7)$ & $54,5(17,2)$ & $50,9(16,9)$ & $51,7(17,1)$ & $50,3(18,1)$ & $45,0(16,2)$ \\
\hline \multicolumn{9}{|l|}{ Curso mais elevado } \\
\hline Nunca frequentou a escola & $2(1,4)$ & $13(10,4)$ & $21(15,2)$ & $17(14,9)$ & $4(11,8)$ & $84(8,2)$ & $9(9,4)$ & $14(10,7)$ \\
\hline Até $4^{\circ}$ série do fundamental & $69(47,6)$ & $61(48,8)$ & $62(44,9)$ & $45(39,5)$ & $17(50)$ & $29(59,2)$ & $35(36,5)$ & $44(33,6)$ \\
\hline Fundamental completo/ incompleto & $28(19,3)$ & $28(18,4)$ & $27(19,6)$ & $22(19,3)$ & $9(26,5)$ & $7(14,3)$ & $24(25)$ & $38(29)$ \\
\hline Médio completo & $40(27,6)$ & $110(17,6)$ & $26(18,8)$ & $24(21,1)$ & $4(11,8)$ & $8(16,3)$ & $20(20,8)$ & $33(25,2)$ \\
\hline Pelo menos 1 ano superior & $6(4,1)$ & $6(4,8)$ & $2(1,4)$ & $6(5,3)$ & $0(0)$ & $1(2)$ & $8(8,3)$ & $2(1,5)$ \\
\hline \multicolumn{9}{|l|}{ Classe social } \\
\hline$A-B$ & $40(27,6)$ & $16(12,8)$ & $19(13,8)$ & $21(18,4)$ & $2(5,9)$ & $4(8,2)$ & $39(40,6)$ & $27(20,6)$ \\
\hline C & $73(50,3)$ & $68(54,4)$ & $74(53,6)$ & $52(45,6)$ & $13(38,2)$ & $21(42,9)$ & $39(40,6)$ & $69(52,7)$ \\
\hline$D-E$ & $32(22,1)$ & $41(32,8)$ & $45(32,6)$ & $41(36)$ & $19(55,9)$ & $24(49)$ & $18(18,8)$ & $35(26,7)$ \\
\hline
\end{tabular}

Legenda: (DP) Desvio padrão. Fonte: Rodrigues CFM, et al., 2020. 
Tabela 2 - Perfil de acesso ao serviço de saúde dos usuários nas unidades de saúde, n=832.

\begin{tabular}{|c|c|c|c|c|c|c|c|c|}
\hline \multirow{3}{*}{ Variável } & \multicolumn{8}{|c|}{ Unidades de Saúde } \\
\hline & $\mathbf{A}$ & B & $\mathbf{C}$ & $\mathbf{D}$ & $\mathbf{E}$ & $\mathbf{F}$ & $\mathbf{G}$ & $\mathbf{H}$ \\
\hline & n (\%) & n (\%) & n (\%) & n (\%) & n (\%) & n (\%) & n (\%) & n (\%) \\
\hline Total & $145(100)$ & $125(100)$ & $138(100)$ & $114(100)$ & $34(100)$ & $49(100)$ & $96(100)$ & $131(100)$ \\
\hline \multicolumn{9}{|c|}{$\begin{array}{l}\text { Domicílio está cadastrado no Programa Saúde da } \\
\qquad \text { Família? }\end{array}$} \\
\hline Sim & $141(97,9)$ & $121(98,4)$ & $133(97,8)$ & $114(100)$ & $33(97,1)$ & $49(100)$ & $89(97,8)$ & $130(99,2)$ \\
\hline \multicolumn{9}{|c|}{$\begin{array}{l}\text { Utiliza ou já utilizou algum serviço de saúde do } \\
\text { SUS? }\end{array}$} \\
\hline Sim & $143(98,6)$ & $124(99,2)$ & $138(100)$ & $114(100)$ & $33(97,1)$ & $49(100)$ & $94(97,9)$ & $129(98,5)$ \\
\hline \multicolumn{9}{|l|}{ Recebe visita domiciliar de ACS? } \\
\hline Sim & $138(95,8)$ & $110(88,7)$ & $127(92)$ & $113(99,1)$ & $31(91,2)$ & $47(95,9)$ & $93(96,9)$ & $99(76,2)$ \\
\hline \multicolumn{9}{|l|}{ Tem algum plano de saúde médico? } \\
\hline Sim & $62(43,1)$ & $43(34,4)$ & $24(17,5)$ & $47(41,2)$ & $9(26,5)$ & $22(44,9)$ & $47(49)$ & $37(28,2)$ \\
\hline \multicolumn{9}{|c|}{ No último ano, realizou alguma consulta médica? } \\
\hline Sim & $127(89,4)$ & $103(86,6)$ & $119(89,5)$ & $99(90)$ & $30(88,2)$ & $43(87,8)$ & $90(93,8)$ & $114(87,7)$ \\
\hline \multicolumn{9}{|l|}{ Esteve internado no último ano? } \\
\hline Sim & $32(22,1)$ & $30(24,2)$ & $36(26,1)$ & $31(27,2)$ & $5(15,2)$ & $12(24,5)$ & $22(22,9)$ & $33(25,2)$ \\
\hline \multicolumn{9}{|l|}{ Serviço mais utilizado? } \\
\hline Atenção Primária à Saúde & $137(94,5)$ & $124(99,2)$ & $138(100)$ & $113(99,1)$ & $32(94,1)$ & $48(98)$ & $94(97,9)$ & $127(96,8)$ \\
\hline CEAE & $122(84,1)$ & $105(84)$ & $120(87)$ & $86(75,4)$ & $28(82,4)$ & $42(85,7)$ & $78(81,3)$ & $108(82,4)$ \\
\hline Hospital & $111(76,6)$ & $107(85,6)$ & $117(84,8)$ & $98(86)$ & $25(73,5)$ & $41(83,7)$ & $80(83,3)$ & $116(88,5)$ \\
\hline Pronto Socorro & $83(57,2)$ & $92(73,6)$ & $111(80,4)$ & $78(68,4)$ & $21(61,8)$ & $35(71,4)$ & $72(75)$ & $101(77,1)$ \\
\hline Laboratório Municipal & $116(6,9)$ & $110(88)$ & $128(92,8)$ & $96(84,2)$ & $27(79,4)$ & $48(98)$ & $84(87,5)$ & $118(90,1)$ \\
\hline Centro de Atenção Psicossocial & $10(6,9)$ & $19(15,2)$ & $27(19,6)$ & $12(10,5)$ & $4(11,8)$ & $6(12,2)$ & $14(14,6)$ & $11(8,4)$ \\
\hline Farmácia & $132(91)$ & $116(92,8)$ & $135(97,8)$ & $107(93,9)$ & $33(97,0)$ & $47(95,9)$ & $84(87,5)$ & $119(90,8)$ \\
\hline Outros & $0(0)$ & $0(0)$ & $0(0)$ & $0(0)$ & $0(0)$ & $0(0)$ & $0(0)$ & $0(0)$ \\
\hline
\end{tabular}

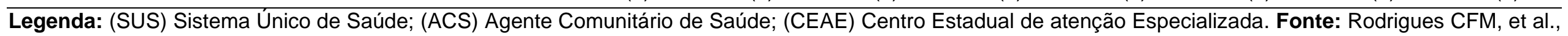
2020. 
Tabela 3 - Características sócio demográficas dos profissionais de saúde, antes da intervenção do MACC (2013), durante (2015) e três anos após (2018).

\begin{tabular}{|c|c|c|c|c|c|c|c|c|c|c|}
\hline \multirow{3}{*}{ Variável } & \multirow{3}{*}{ Fase } & \multicolumn{9}{|c|}{ Unidades de Saúde } \\
\hline & & A & B & C & D & $E$ & $\mathbf{F}$ & G & $\mathbf{H}$ & I \\
\hline & & n (\%) & n (\%) & n (\%) & n (\%) & n (\%) & n (\%) & n (\%) & n (\%) & n (\%) \\
\hline \multicolumn{11}{|l|}{ Sexo } \\
\hline & 2013 & $12(92,3)$ & $6(100)$ & $13(100)$ & $9(81,8)$ & $10(90,9)$ & $10(100)$ & $13(100)$ & $7(100)$ & $10(90,9)$ \\
\hline \multirow[t]{2}{*}{ Feminino } & 2015 & $9(100)$ & $6(85,7)$ & $5(83,3)$ & $7(100)$ & $6(75)$ & $7(100)$ & $7(100)$ & $7(100)$ & $5(100)$ \\
\hline & 2018 & $9(90)$ & $10(100)$ & $8(100)$ & $10(100)$ & $8(80)$ & $8(80)$ & $9(90)$ & $9(90)$ & $4(100)$ \\
\hline \multicolumn{11}{|l|}{ Idade } \\
\hline \multirow{3}{*}{ Média ( $( \pm D P)$} & 2013 & $40(11)$ & $31(9)$ & $40(12)$ & $43(12)$ & $37(12)$ & $33(8)$ & $46(14)$ & $34(11)$ & $31(10)$ \\
\hline & 2015 & $47(10)$ & $32(10)$ & $36(11)$ & $45(12)$ & $30(12)$ & $32(8)$ & $41(12)$ & 34 (13) & $29(2)$ \\
\hline & 2018 & $43(13)$ & $41(11)$ & $33(8)$ & $43(11)$ & $34(11)$ & $35(7)$ & $39(14)$ & $35(11)$ & $29(3)$ \\
\hline \multicolumn{11}{|l|}{ Escolaridade } \\
\hline \multirow{3}{*}{ Ensino médio } & 2013 & $10(76,9)$ & $3(50)$ & $8(61,5)$ & $5(50)$ & $5(50)$ & $2(33,3)$ & $7(70)$ & $5(71,4)$ & $1(9,1)$ \\
\hline & 2015 & $7(77,8)$ & $4(57,1)$ & $4(66,7)$ & $5(71,4)$ & $6(75)$ & $4(57,1)$ & $5(71,4)$ & $6(85,7)$ & $1(20)$ \\
\hline & 2018 & $4(40)$ & $8(80)$ & $3(37,5)$ & $7(70)$ & $5(50)$ & $5(50)$ & $8(80)$ & $5(50)$ & $0(0)$ \\
\hline \multirow{3}{*}{ Ensino superior e pós Graduação } & 2013 & $2(15,4)$ & $2(33,3)$ & $4(30,8)$ & $4(40)$ & $3(30)$ & $3(50)$ & $3(30)$ & $1(14,3)$ & $10(90,9)$ \\
\hline & 2015 & $1(11,1)$ & $3(42,9)$ & $1(33,4)$ & $1(14,3)$ & $2(25)$ & $3(42,9)$ & $2(28,6)$ & $0(0)$ & $4(80)$ \\
\hline & 2018 & $0(0)$ & $1(10)$ & $4(50)$ & $3(30)$ & $5(50)$ & $5(50)$ & $2(20)$ & $4(40)$ & $4(100)$ \\
\hline \multicolumn{11}{|l|}{ Tempo atuação no serviço } \\
\hline \multirow{3}{*}{6 ou mais anos } & 2013 & $3(23,1)$ & $1(16,7)$ & $4(30,7)$ & $2(20)$ & $4(36,4)$ & $5(50)$ & $3(23,1)$ & $2(28,6)$ & $2(20)$ \\
\hline & 2015 & $4(44,4)$ & $1(14,3)$ & $2(33,3)$ & $5(71,4)$ & $3(37,5)$ & $2(28,6)$ & $2(28,6)$ & $2(28,6)$ & $2(40)$ \\
\hline & 2018 & $5(50)$ & $4(40)$ & $3(37,5)$ & $6(60)$ & $2(20)$ & $5(50)$ & $4(40)$ & $3(30)$ & $2(50)$ \\
\hline
\end{tabular}

Legenda: (DP) Desvio padrão. Fonte: Rodrigues CFM, et al., 2020. 


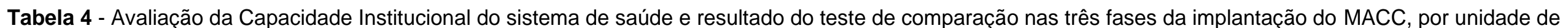
saúde, $\mathrm{n}=9$.

\begin{tabular}{|c|c|c|c|c|c|c|c|c|c|c|}
\hline $\begin{array}{l}\text { Unidades } \\
\text { de Saúde }\end{array}$ & Fase & D 1 & $\begin{array}{c}\text { Mediana } \\
\text { D } 2\end{array}$ & $\begin{array}{c}\text { los escc } \\
\text { D } 3\end{array}$ & $\begin{array}{c}\text { es por c } \\
\text { D } 4\end{array}$ & $\begin{array}{c}\text { mensõe } \\
\text { D } 5\end{array}$ & $\begin{array}{c}\text { do ACl } \\
\text { D } 6\end{array}$ & D 7 & Avaliação Global & Capacidade Institucional para Condições Crônicas \\
\hline A & $\begin{array}{l}2013 \\
2015 \\
2018\end{array}$ & $\begin{array}{l}6,83 \\
8,83 \\
8,42\end{array}$ & $\begin{array}{l}5,25 \\
7,50 \\
3,50\end{array}$ & $\begin{array}{c}5,75 \\
10,00 \\
7,25\end{array}$ & $\begin{array}{l}4,50 \\
9,50 \\
7,63\end{array}$ & $\begin{array}{c}6,83 \\
10,00 \\
10,25\end{array}$ & $\begin{array}{c}2,50 \\
8,17 \\
10,17\end{array}$ & $\begin{array}{l}2,17 \\
7,83 \\
7,67\end{array}$ & $\begin{array}{l}4,89 \\
8,83 \\
7,67\end{array}$ & $\begin{array}{l}\text { Capacidade básica } \\
\text { Capacidade razoável } \\
\text { Capacidade razoável }\end{array}$ \\
\hline p-valor & & $<0,001$ & $<0,001$ & $<0,001$ & $<0,001$ & $<0,001$ & $<0,001$ & $<0,001$ & $<0,001$ & \\
\hline B & $\begin{array}{l}2013 \\
2015 \\
2018\end{array}$ & $\begin{array}{l}5,08 \\
7,83 \\
9,17\end{array}$ & $\begin{array}{l}3,25 \\
6,25 \\
7,75\end{array}$ & $\begin{array}{l}5,63 \\
7,50 \\
9,63\end{array}$ & $\begin{array}{l}5,25 \\
9,00 \\
8,75\end{array}$ & $\begin{array}{l}5,92 \\
9,00 \\
9,50\end{array}$ & $\begin{array}{l}5,58 \\
7,17 \\
9,42\end{array}$ & $\begin{array}{l}3,83 \\
6,00 \\
8,00\end{array}$ & $\begin{array}{l}5,03 \\
7,58 \\
8,82\end{array}$ & $\begin{array}{l}\text { Capacidade básica } \\
\text { Capacidade razoável } \\
\text { Capacidade razoável }\end{array}$ \\
\hline p-valor & & $<0,001$ & 0,001 & $<0,001$ & 0,001 & 0,001 & $<0,001$ & $<0,001$ & $<0,001$ & \\
\hline C & $\begin{array}{l}2013 \\
2015 \\
2018\end{array}$ & $\begin{array}{l}4,67 \\
6,58 \\
9,83\end{array}$ & $\begin{array}{c}4,50 \\
4,00 \\
10,00\end{array}$ & $\begin{array}{c}4,00 \\
6,25 \\
10,00\end{array}$ & $\begin{array}{c}5,75 \\
4,25 \\
10,00\end{array}$ & $\begin{array}{c}8,00 \\
8,08 \\
10,17\end{array}$ & $\begin{array}{c}4,50 \\
4,08 \\
10,00\end{array}$ & $\begin{array}{c}4,17 \\
2,58 \\
10,00\end{array}$ & $\begin{array}{l}4,93 \\
5,10 \\
9,99\end{array}$ & $\begin{array}{l}\text { Capacidade básica } \\
\text { Capacidade básica } \\
\text { Capacidade ótima }\end{array}$ \\
\hline p-valor & & $<0,001$ & $<0,001$ & $<0,001$ & $<0,001$ & $<0,001$ & $<0,001$ & $<0,001$ & $<0,001$ & \\
\hline D & $\begin{array}{l}2013 \\
2015 \\
2018 \\
\end{array}$ & $\begin{array}{l}8,00 \\
8,33 \\
8,58\end{array}$ & $\begin{array}{l}6,75 \\
8,25 \\
6,17\end{array}$ & $\begin{array}{l}8,00 \\
8,25 \\
9,50\end{array}$ & $\begin{array}{l}4,50 \\
7,00 \\
7,63\end{array}$ & $\begin{array}{l}8,83 \\
9,33 \\
9,17\end{array}$ & $\begin{array}{l}5,50 \\
6,33 \\
8,25\end{array}$ & $\begin{array}{l}5,33 \\
6,33 \\
7,25\end{array}$ & $\begin{array}{l}6,69 \\
7,74 \\
7,97\end{array}$ & $\begin{array}{l}\text { Capacidade razoável } \\
\text { Capacidade razoável } \\
\text { Capacidade razoável }\end{array}$ \\
\hline p-valor & & 0,001 & $<0,001$ & 0,001 & $<0,001$ & 0,019 & $<0,001$ & $<0,001$ & $<0,001$ & \\
\hline E & $\begin{array}{l}2013 \\
2015 \\
2018\end{array}$ & $\begin{array}{l}7,67 \\
9,33 \\
8,92\end{array}$ & $\begin{array}{l}5,00 \\
7,75 \\
8,00\end{array}$ & $\begin{array}{l}7,00 \\
9,25 \\
9,75\end{array}$ & $\begin{array}{c}7,50 \\
9,63 \\
10,13 \\
\end{array}$ & $\begin{array}{c}7,17 \\
9,42 \\
10,08\end{array}$ & $\begin{array}{l}3,33 \\
8,17 \\
9,33\end{array}$ & $\begin{array}{l}2,33 \\
7,92 \\
8,92\end{array}$ & $\begin{array}{l}5,54 \\
8,77 \\
9,32\end{array}$ & $\begin{array}{l}\text { Capacidade básica } \\
\text { Capacidade razoável } \\
\text { Capacidade ótima }\end{array}$ \\
\hline p-valor & & $<0,001$ & $<0,001$ & $<0,001$ & $<0,001$ & $<0,001$ & $<0,001$ & $<0,001$ & $<0,001$ & \\
\hline$F$ & $\begin{array}{l}2013 \\
2015 \\
2018 \\
\end{array}$ & $\begin{array}{c}8,17 \\
4,00 \\
10,33 \\
\end{array}$ & $\begin{array}{l}7,63 \\
2,00 \\
8,00\end{array}$ & $\begin{array}{l}6,75 \\
3,75 \\
9,88\end{array}$ & $\begin{array}{l}4,63 \\
3,00 \\
9,50\end{array}$ & $\begin{array}{c}8,75 \\
5,83 \\
10,58 \\
\end{array}$ & $\begin{array}{c}4,50 \\
6,33 \\
10,50\end{array}$ & $\begin{array}{l}4,50 \\
4,50 \\
8,67\end{array}$ & $\begin{array}{l}6,44 \\
4,04 \\
9,60\end{array}$ & $\begin{array}{l}\text { Capacidade razoável } \\
\text { Capacidade básica } \\
\text { Capacidade ótima }\end{array}$ \\
\hline p-valor & & $<0,001$ & $<0,001$ & $<0,001$ & $<0,001$ & $<0,001$ & $<0,001$ & $<0,001$ & $<0,001$ & \\
\hline $\mathbf{G}$ & $\begin{array}{l}2013 \\
2015 \\
2018\end{array}$ & $\begin{array}{l}4,33 \\
4,33 \\
9,92\end{array}$ & $\begin{array}{l}5,25 \\
3,75 \\
7,75\end{array}$ & $\begin{array}{l}5,00 \\
5,25 \\
9,50\end{array}$ & $\begin{array}{l}2,75 \\
7,25 \\
9,88\end{array}$ & $\begin{array}{c}6,60 \\
9,67 \\
10,17\end{array}$ & $\begin{array}{c}5,00 \\
7,83 \\
10,58 \\
\end{array}$ & $\begin{array}{l}3,17 \\
7,33 \\
9,25\end{array}$ & $\begin{array}{l}4,63 \\
6,42 \\
9,64\end{array}$ & $\begin{array}{l}\text { Capacidade básica } \\
\text { Capacidade razoável } \\
\text { Capacidade ótima }\end{array}$ \\
\hline p-valor & & $<0,001$ & $<0,001$ & $<0,001$ & $<0,001$ & $<0,001$ & $<0,001$ & $<0,001$ & $<0,001$ & \\
\hline $\mathbf{H}$ & $\begin{array}{l}2013 \\
2015 \\
2018\end{array}$ & $\begin{array}{l}6,67 \\
9,00 \\
9,92\end{array}$ & $\begin{array}{l}6,25 \\
9,00 \\
9,25\end{array}$ & $\begin{array}{c}7,50 \\
9,25 \\
10,13\end{array}$ & $\begin{array}{l}3,00 \\
9,25 \\
9,75\end{array}$ & $\begin{array}{c}6,00 \\
9,50 \\
10,08\end{array}$ & $\begin{array}{c}3,67 \\
7,50 \\
10,50\end{array}$ & $\begin{array}{l}2,17 \\
7,50 \\
8,42\end{array}$ & $\begin{array}{l}5,17 \\
8,67 \\
9,83\end{array}$ & $\begin{array}{l}\text { Capacidade básica } \\
\text { Capacidade razoável } \\
\text { Capacidade ótima }\end{array}$ \\
\hline p-valor & & $<0,001$ & 0,002 & $<0,001$ & $<0,001$ & $<0,001$ & $<0,001$ & $<0,001$ & $<0,001$ & \\
\hline I & $\begin{array}{l}2013 \\
2015 \\
2018 \\
\end{array}$ & $\begin{array}{c}3,33 \\
9,7 \\
10,83 \\
\end{array}$ & $\begin{array}{c}5,75 \\
10,00 \\
10,50 \\
\end{array}$ & $\begin{array}{l}10,00 \\
11,00 \\
11,00 \\
\end{array}$ & $\begin{array}{l}10,00 \\
10,75 \\
11,00 \\
\end{array}$ & $\begin{array}{l}10,17 \\
10,67 \\
11,00 \\
\end{array}$ & $\begin{array}{c}6,17 \\
10,50 \\
10,00 \\
\end{array}$ & $\begin{array}{l}10,00 \\
10,50 \\
10,00 \\
\end{array}$ & $\begin{array}{c}7,93 \\
10,40 \\
10,60 \\
\end{array}$ & $\begin{array}{c}\text { Capacidade razoável } \\
\text { Capacidade ótima } \\
\text { Capacidade ótima }\end{array}$ \\
\hline
\end{tabular}

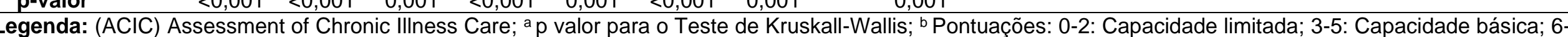
8: Capacidade razoável; 9-11: capacidade ótima. Fonte: Rodrigues CFM, et al., 2020. 


\section{DISCUSSÃO}

Os resultados do presente estudo apontam uma evolução positiva e significativa na capacidade institucional para atenção as CC de saúde após intervenção por meio do MACC em todas as unidades de saúde do município de Santo Antônio do Monte. Tal evolução foi observada em todas as dimensões do ACIC. Ao analisar os resultados por unidade de saúde, observa-se que a maioria melhorou sua capacidade para atuação nas CC passando de básica e razoável para ótima.

Estes resultados positivos da implementação do MACC, são de grande importância para atender ao atual cenário de CC de forma proativa, contínua e integrada (MENDES EV, 2010). A experiência de Santo Antônio do Monte reportou não apenas uma melhoria na capacidade institucional, como também a possibilidade de sustentar esses resultados no médio prazo, mesmo em um contexto de rotatividade de profissionais. Além disso, as mudanças são percebidas seis anos depois do início da intervenção, quando o município já apresenta total autonomia no gerenciamento do MACC.

Em relação ao perfil do acesso e dos usuários que frequentam as unidades de saúde observou-se que a APS é o serviço mais utilizado demonstrando a importância do SUS mesmo em um município de IDH médio e com elevada cobertura do sistema privado de saúde.

Este resultado vai de encontro aos princípios da RAS no que tange à APS como ordenadora e porta de entrada preferencial do sistema de saúde (MENDES EV, 2011). Sistemas de saúde organizados a partir da APS propiciam melhor acompanhamento da população, evitando agravamento das doenças, e a consequente sobrecarga dos demais níveis de atenção (ROCHA TAH e SILVA NC, 2019).

Outro achado importante desta investigação foi o tempo de atuação dos profissionais. Em $50 \%$ das unidades de saúde os profissionais atuam seis anos ou mais. Sabe-se que quanto maior o tempo na unidade maior será o vínculo profissional e usuário, essencial na APS para garantir a longitudinalidade do cuidado ofertado (STARFIELD B, 2002). Evidências apontam que a continuidade do cuidado é capaz de reduzir internações e o uso de serviços de emergência (KRINGOS DS, et al., 2010).

Quanto à Capacidade Institucional, todas as unidades, exceto a UBS D, apresentaram evolução importante para gerenciar o MACC, obtendo classificação ótima em 2018 na percepção dos profissionais. A unidade de saúde "D", manteve-se com avaliação de Capacidade razoável nos três anos de avaliação. Observa-se que esta unidade de saúde D já começou com uma mediana maior na linha de base, em 2013, quando comparada com as demais unidades.

Os resultados observados em Santo Antônio do Monte contrastam com outras experiências no Brasil. Em Curitiba (PR), utilizando o mesmo instrumento dessa investigação, não foi identificada diferença estatisticamente significativa na capacidade institucional para gerenciamento das CC. Porém, essa intervenção ocorreu em apenas uma UBS e a avaliação foi conduzida após um ano de implantação do MACC (SCHWAB GL, et al., 2014).

Vale ressaltar que melhorias observadas em Santo Antônio do Monte ocorreram mesmo em um contexto político e laboral pouco favorável. Esse período se caracterizou por instabilidade política com a renúncia do prefeito e por alteração importante no quadro de funcionários devido a alteração na forma de contratação dos profissionais.

Esses fatores certamente impactaram o processo de implementação do MACC no município. A intervenção estava pactuada com os atores chaves que foram, em sua maioria, alterados no processo. A mudança proposta nos micros e macroprocessos da APS foi absorvida pelos novos profissionais dando continuidade à consolidação do modelo no município.

A análise por dimensão observou um aumento expressivo no escore das dimensões 6 (Sistema de Informação Clínica) e 7 (Integração dos Componentes do Modelo de Atenção as Condições Crônicas). 0 aumento da Dimensão 6 (Sistema de Informação Clínica) pode estar relacionado à efetiva implantação do prontuário eletrônico em 2017 nas unidades de saúde, permitindo assim a integração do cuidado entre os diferentes níveis da rede de atenção. 
Em estudo realizado em Campo Grande, Mato Grosso do Sul, utilizando o mesmo instrumento na linha de base do MACC observou-se que a dimensão "Sistema de Informação Clínica" foi a principal fragilidade e o "Desenho do Sistema de Prestação de Serviço" a de maior potencial (COSTA KC, et al., 2016). Apesar dos achados semelhantes entre Samonte e Campo Grande estes possuem características muito distintas tanto em relação ao porte populacional quanto no recorte da avaliação nos serviços de atenção primária. Campo Grande é um município de grande porte, com aproximadamente 900 mil habitantes e a avaliação da capacidade institucional foi realizada em cinco equipe de atenção primária e não em todos os serviços de atenção primária do município, além de ser uma avaliação seccional e não longitudinal como Samonte.

Apesar das características operacionais distintas sabe-se da dificuldade de implantar prontuário eletrônico em saúde e informatização de serviços de saúde tanto em municípios pequenos como maiores, mesmo com a premissa de que a implantação de prontuário eletrônico melhora a coordenação e continuidade do cuidado e a comunicação e monitoramento de indicadores clínicos (STARFIELD B, 2002; DIAS MP e GIOVANELLA L, 2013; GONÇALVES JPP, et al., 2013; COSTA JFR e PORTELA MC, 2018). Em relação a Dimensão 7 (Integração dos componentes do MACC), a melhora observada em Samonte pode estar relacionada à forma de implementação do modelo no município ocorreu de forma ampla no sistema de saúde do município, abrangendo todas as unidades básicas de saúde, além do centro de especialidades e não somente em unidades piloto. Essa abrangência facilitou a percepção da organização de um sistema de governança da rede pelos profissionais.

Em relação às dimensões que apresentaram menor evolução, destaca-se a Dimensão 2 (Articulação com a Comunidade). A articulação com a comunidade é um ponto crítico na implantação do MACC, uma vez que para se obter resultados efetivos faz-se necessário que usuários sejam responsáveis e ativos no autocuidado sendo preparados e imponderados pela equipe de saúde sobre sua saúde, tendo uma atitude proativa (WAGNER EH, 1998). Apesar de não ser uma tarefa fácil estabelecer mudanças nos processos de trabalho, principalmente no cuidado em saúde onde os profissionais tem muita autonomia, os achados desta pesquisa demonstram a viabilidade de implementação do MACC de forma sustentável em sistemas locais de saúde. Uma ferramenta importante na mudança de processos de trabalho é a autoavaliação dos próprios profissionais que estão à frente das dificuldades e desafios diários nas unidades de saúde.

Neste contexto, um aspecto que pode ter colaborado na sustentabilidade dos resultados do MACC foi a presença do Programa Nacional de Melhoria do Acesso e da Qualidade da Atenção Básica (PMAQ), que monitorou os macroprocessos do MACC. O $3^{\circ}$ ciclo do PMAQ iniciou-se em 2015, com avaliação externa no segundo semestre de 2017, realizando nesse período, acompanhamento e avaliação do trabalho das equipes de saúde (BRASIL, 2017). Em Santo Antônio do Monte, das oito equipes de APS avaliadas, quatro observaram um resultado muito bom $(B, C, D$ e H), três bons $(A, F$ e $G)$ e uma regular ( $E)$.

Para que a RAS seja efetiva é de suma importância que existem mecanismos de governança da rede implantados, ou seja, processos de tomada de decisão, mobilização e uso de recursos, de forma a implementar e avaliar políticas que influenciam a saúde, o manejo dos conflitos de interesse, e a progressiva estabilização da rede baseada em resultados (BRASIL, 2016). Esse processo precisa ser aprimorado no contexto municipal e regional com foco na melhoria do cuidado em saúde ofertado e, consequentemente, da percepção do profissional de saúde da capacidade institucional para atenção às $C C$ em redes.

\section{CONCLUSÃO}

Os resultados da presente investigação mostraram uma significativa evolução da capacidade institucional do sistema de saúde para o cuidado às Condição Crônicas; passando de razoável e básica para ótima após a intervenção do MACC, refletindo no fortalecimento do sistema local de saúde. Dentre as unidades de saúde avaliadas, apenas uma não apresentou mudança na capacidade institucional, apesar do aumento no escore de Capacidade Institucional. Estes achados podem subsidiar outros municípios, principalmente aqueles com realidade semelhante, na organização da rede de atenção à saúde e consequentemente ter melhoria do cuidado às Condição Crônicas. Espera-se que este fortalecimento do sistema de saúde para atuação nas CC evidenciados nessa investigação seja sustentável ao longo do tempo e que seu impacto reflita de forma direta nos indicadores de saúde da população. 


\section{AGRADECIMENTOS E FINANCIAMENTO}

Financiamento: Organização Pan-americana da Saúde (OPAS), Carta Acordo OPAS - IPEAD / FACE / UFMG BR / LOA / 1400085.001; Conselho Nacional dos Secretários de Saúde (CONASS). Agradecimento: Fapemig pelo Auxilio Financeiro recebido através do Projeto Pesquisador Mineiro e do Projeto de Pesquisa para o Sistema Unico de Saúde - Fapemig Edital 14/2013 Pesquisa para o SUS CSA - APQ-03559-13.

\section{REFERÊNCIAS}

1. ABEGUNDE DO, et al. The burden and costs of chronic diseases in low-income and middle-income countries. Lancet, 2007; 370(9603): 1929-1938.

2. ANDRADE MV, et al. Challenges and lessons from a primary care intervention in a Brazilian municipality. Revista de Saúde Pública, 2019; 53(45): 1-11.

3. BAPTISTA DR, et al. The chronic care model for type 2 diabetes: a systematic review. Diabetology \& Metabolic Syndrome, 2016; 8(7): 1-7.

4. BRASIL. Agência Nacional de Saúde Suplementar. Beneficiários de planos privados de saúde, por cobertura assistencial (Brasil - 2010-2020). - Rio de Janeiro: Agência Nacional de Saúde Suplementar, 2020. Disponível em: https://www.ans.gov.br/perfil-do-setor/dados-gerais. Acesso em: 22 jul 2020.

5. BRASIL. Conselho Nacional de Secretários de Saúde. Governança Regional das Redes de Atenção à Saúde / Conselho Nacional de Secretários de Saúde. - Brasília: CONASS, 2016. 118 p.

6. BRASIL. Ministério da Saúde. Secretaria de Atenção Primária à Saúde. Programa de Melhoria do Acesso e Qualidade / Ministério da Saúde. Secretaria de Atenção Primária à Saúde. - Brasília: Ministério da Saúde, 2017. Disponível em: https://aps.saude.gov.br/ape/pmaq. Acesso em: 22 jul 2020.

7. COSTA JFR, PORTELA MC. Percepciones de gestores, profesionales y usuarios acerca del registro electrónico de salud y de aspectos facilitadores y barreras para su implementación. Cadernos de Saúde Pública, 2018; 34(1): e00187916.

8. COSTA KC, et al. Assessment of Chronic Illness Care (ACIC): avaliação da aplicabilidade e resultados. Saúde Debate, 2016; 40(108): 106-117.

9. D'ÁVILA ALV, et al. Regionalização e Redes de Saúde. Ciência \& Saúde Coletiva, 2018; 23(6): 1791-1798.

10. DAVY C, et al. Effectiveness of chronic care models: opportunities for improving healthcare practice and health outcomes: a systematic review. BMC Health Services Research, 2015; 15(194): 1-11.

11. DIAS MP, GIOVANELLA L. Prontuário eletrônico - uma estratégia de coordenação entre a atenção primária e secundária à saúde no município de Belo Horizonte. Revista Eletrônica de Comunicação, Informação \& Inovação em Saúde, 2013; 7(2): 1-17.

12. EVANGELISTA MJO, et al. Planning and building Health care Networks in Brazil's Federal District. Ciência \& Saúde Coletiva, 2019; 24(6): 2115-2124.

13. GILL P, et al. Methods of data collection in qualitative research: Interviews and focus groups. British Dental Journal, 2008; 204(6): 291-295.

14. GONÇALVES JPP, et al. Prontuário Eletrônico: uma ferramenta que pode contribuir para a integração das Redes de Atenção à Saúde. Saúde Debate, 2013; 37(96): 43-50.

15. INSTITUTO BRASILEIRO DE GEOGRAFIA E ESTATÍSTICA. 2019. Cidades (Santo Antônio do Monte - Minas Gerais). Brasil: IBGE. Disponível em: https://cidades.ibge.gov.br/brasil/mg/santo-antonio-do-monte/panorama. Acesso em: 21 jul. 2020.

16. KRINGOS DS, et al. The breadth of primary care: a systematic literature review of its core dimensions. BMC Health Services Research, 2010; 10(65): 1-13.

17. MALTA DC, et al. Noncommunicable diseases and the use of health services: analysis of the National Health Survey in Brazil. Revista de Saúde Pública, 2017; 51(Supl 1): 1-10.

18. MENDES EV, et al. Integrated care in the unified health system of Brazil: The laboratory for innovation in chronic conditions in Santo Antônio do Monte. International Journal of Healthcare Management, 2018; 12(2): 116-122.

19. MENDES EV. As Redes de Atenção à Saúde. 2nd ed. Brasília: Organização Pan-Americana da Saúde, $2011 ; 549 p$.

20. MENDES EV. As Redes de Atenção à saúde. Ciência \& Saúde Coletiva, 2010; 15(5): 2297-2305.

21. MOYSÉS ST, et al. Laboratório de Inovações no cuidado das Condições Crônicas na APS: A implantação do modelo de atenção às condições crônicas na UBS Alvorada em Curitiba, Paraná. Brasília: Organização Pan-Americana da Saúde, 2012.

22. ROBUSTO F, et al. The effects of the introduction of a chronic care model-based program on utilization of healthcare resources: the results of the Puglia care program. BMC Health Services Research, 2018; 18(377): 1-25.

23. ROCHA TAH, SILVA NC. Oferta de cuidado primário para doenças crônicas: uma análise da eficiência técnicas das equipes de saúde brasileiras. APS em Revista, 2019; 1(1): 18-28.

24. SCHMIDT MI, et al. Chronic non-communicable diseases in Brazil: Burden and current challenges. Lancet, 2011; 377(9781): 1949-1961.

25. SCHRAMM JMA, et al. Epidemiological transition and the study of burden of disease in Brazil. Ciência \& Saúde Coletiva, 2004; 9(4): 897-908.

26. SCHWAB GL, et al. Percepção de inovações na atenção às doenças/condições crônicas: uma pesquisa avaliativa em Curitiba. Saúde Debate, 2014; 38(special): 307-318.

27. STARFIELD B. Atenção primária: equilíbrio entre necessidades de saúde, serviços e tecnologia. Brasília: UNESCO, 2002; 726p.

28. WAGNER $\mathrm{EH}$. Chronic disease management: what will take to improve care for chronic illness. Effective Clinical Practice, 1998; 1(1): 2-4. 